expected magnitude and scaling. For example, do they decrease with increasing plasma current? As this loss mechanism is directly germane to future reactors, it is especially important to check the theoretical predictions at TFTR.

An entirely different genre of $\alpha$-loss mechanisms involves the interaction between coherent magnetohydrodynamic oscillations ('collective' oscillations) and the $\alpha$ s. Like Proteus, these oscillations can manifest themselves in different guises, as 'fishbones,' 'snakes' or 'sharkteeth', to name but a few, and if they grow large enough they can eject energetic particles, such as the heating beam ions or the $\alpha$ s, from the plasma (B. Coppi, Massachusetts Institute of Technology). At high concentrations (about 1 per cent), the $\alpha$ s themselves can drive these instabilities (unlikely in TFTR, but possible in future reactors).

The chosen operating parameters for TFTR kept these collective modes to a minimum, and for these levels there were no associated $\alpha$ losses (H. Hsuan, PPPL; and L. Zakharov, PPPL). But the discharge with maximum fusion power may have been affected - one school of thought attributes the droop in fusion power, at least in part, to a coherent mode that appeared just after the peak.

One collective oscillation that could be a particular problem in future machines is the $\alpha$-driven Alfvén mode (H. Kimura, Japanese Atomic Energy Research Institute; C. T. Hsu, Massachusetts Institute of Technology). Amongst other conditions, this instability occurs when the average $\alpha$ velocity $\left(1.3 \times 10^{7} \mathrm{~m} \mathrm{~s}^{-1}\right.$ at birth $)$ is higher than the Alfvén velocity $\left(v_{\mathrm{A}}=B /\right.$ $\sqrt{4 \pi m_{\mathrm{i}} n_{\mathrm{i}}}$, where $B$ is magnetic field and $m_{\mathrm{i}}, n_{\mathrm{i}}$ the mass and number density of the ions). The good news is that it was not observed in the TFTR experiments (E. Fredrickson, PPPL; and R. Nazikian, PPPL), as indeed theoretical calculations had indicated. The ratio of $\alpha$ pressure (the product of number density, $n_{\alpha}$, and kinetic energy, $E_{\alpha}$ ) to magnetic field pressure $\left(B^{2} / 8 \pi \approx 100 \mathrm{~atm}\right)$ would need to be roughly 5 times greater than it typically was in the TFTR (C. Z. Chang, PPPL). But because the conditions are far more likely to be satisfied in future machines, efforts are being made to push the TFTR over the excitation threshold. One way that has been suggested is to drive a larger fraction of the plasma current off-axis (D. Spong, Oak Ridge National Laboratory).

And what about the $\alpha$ s that are well confined, which make up about 90 per cent of those born in the TFTR? As the $\alpha$ heating effects are only a few per cent, can we directly detect them through sensitive $\alpha$-particle diagnostics? In fact, diagnostic methods are being developed that, for example, determine the density of the $\alpha$ s through their elastic collisions with plasma tritons. Some of these energy-boosted tritons, known as knock-ons, then fuse with deuterons generating neutrons that have an energy up to $20.7 \mathrm{MeV}$. To detect these high-energy neutrons, and to avoid being swamped by the $14.1-\mathrm{MeV}$ neutrons from the background $\mathrm{D}-\mathrm{T}$ reaction, an energy threshold detector is to be used: based on a threshold nuclear reaction in beryllium, it is sensitive only to neutrons with energies above $16.5 \mathrm{MeV}$ (R. Fisher, General Atomics, San Diego).

A further important element of the deuterium-tritium experiments has been the study of tritium transport itself. Rather than adding a high proportion of tritium, the essence of this experimental programme has been to inject trace quantities $(\sim 1$ per cent) of tritium into deuterium plasma, either in the energetic heating beams (D. Jassby, PPPL), or by introducing neutral (cold) tritium gas at the plasma edge (P. Efthimion, PPPL).

In the latter case, the inflow and on-axis peaking of the tritons can be observed by imaging the emission of the $14.1-\mathrm{MeV}$ neutrons produced in $\mathrm{D}-\mathrm{T}$ fusion (Fig. 2). The indications are that trace tritium diffuses into the plasma core at about the same rate as trace helium. (Trace helium injections can assist our understanding of the transport of spent $\alpha \mathrm{s}$.) Both tritium and deuterium transport are known to be of great importance in sustaining the fusion power. In fact, it has been suggested that part of the decline in fusion rate (Fig. 1, top trace) may be due to an increased inflow of cold deuterium from the plasma edge (C. Skinner, PPPL), a process known as enhanced recycling.

And what happens to the 'ash' of spent $\alpha s$ ? For a future machine in which $\alpha$ heating dominates over all other forms, might the ash accumulate in the plasma core, diluting the fusion fuel and quenching the fusion burn? Before the TFTR ceases operations this autumn, experiments currently under way should detect the small quantities of $\alpha$ ash within the machine, giving us our first glimpse of this transport (E. Synakowski, PPPL).

So what is this future machine? Given the TFTR's encouraging evidence that the $\alpha$ s did not abscond and that the energy confinement improved, the next logical scientific step is a device in which the $\alpha$-heating is substantial or even dominant, and for which instabilities, either driven or possibly suppressed by the $\alpha$ s, can be readily studied. Will the $\alpha$ s then abscond, or instead heat the plasma?

Richard D. Petrasso is at the Plasma Fusion Center, Massachusetts Institute of Technology, Cambridge, Massachusetts 02139, USA.

Petrasso. R. D. Nature 350. 661 (1991)

Zweben, S. J.etal. Nucl. Fus. 31, 2291 (1991)

Boivin, R. L. et al. Nucl. Fus. 33, 449 (1993).

Goldston. R. J. etal Phys. Rev. Lett. 47.647 (1981)

5. Duperrex, P. A. et al. Nucl. Fus. 32, 1161 (1992).

\section{Dilute pleasure}

ADDICTIVE drugs owe their market not to the pleasure of using them, but to the pain of giving them up. Their withdrawal symptoms are so unpleasant that addicts will commit any crime to get more drug.

So Daedalus wants to alleviate

withdrawal. He recalls the traditional

French alcoholic, who sips wine all day and much of the night. His blood-alcohol never drops to zero, so he never has a hangover. If cocaine and heroin could be sustained like this, instead of giving a brief rush of pleasure followed by hideous withdrawal pains, they would be less of a menace.

Many modern pharmaceuticals can be injected as a subcutaneous fatty 'depot', from which they leach out slowly into the patient's bloodstream. DREADCO's biochemists are nov: devising a blank, rechargeable depot. It will act as an internal buffer for addictive drugs. Most of them, such as amphetamines, cocaine and heroin, are nitrogen bases; they can combine reversibly with acids. So the new drug buffer is an inert fatty polymer with many acidic groupings. It will seize any drug that gets into the user's bloodstream, and slowly release it again. This equilibrium will need careful tuning. The buffer must absorb drug from the blood in levels much above the desired value, and feed it out again to stop the level dropping much below.

When perfected, DREADCO's 'Drugstat' will simply be injected into the addicteither as treatment or as part of the punishment for drug-related crime. His next dose will be a strangely muted experience. There will be no sudden rush of pleasure; as fast as he takes in the drug, the Drugstat depot inside him will seize it. The low equilibrium dose in his bloodstream will give him only a mild mental alleviation. Yet this alleviation will persist wonderfully. Drugstat will maintain the defined concentration in his blood, leaking out just enough drug to avoid withdrawal symptoms. Many days may pass before he has to repeat the dose.

Drugstat will calm the wild roller coaster of the addict's life. It will meter his drug so efficiently that he will need far less of it. His new mental stability may even let him hold down a job and buy his reduced dose without resorting to crime. Furthermore, the pallid, diluted pleasure of Drugstatted addiction will no longer antagonize the respectable masses. Their puritanical envy and outrage at people who get pleasure without working for it, is the basic reason why drugs are illegal in the first place. Once Drugstat is in wide use, public opinion may at last permit drugs to be legalized, thus solving the whole problem. David Jones 